\title{
A New Current Vector Control Method of IPMSM in Flux Weakening Region to Prevent Inverter Voltage Saturation
}

\author{
Yoshihiro Okuyama* Member, Tomohiro Tanaka* Non-member \\ Tsuyoshi Hanamoto** Member, Hiroaki Yamada** Member
}

(Manuscript received Nov. 27, 2012, revised June 10, 2013)

\begin{abstract}
This paper proposes a new current vector control method that prevents voltage saturation by regulating the rate of pulse width modulation (PWM) in a flux weakening region according to a reference. While conventional methods use current limiting on both axes (d-axis and q-axis), our method stabilizes the current vector at onset to and within the flux weakening region using only q-axis current signal control. By eliminating the control of the d-axis current, the circuitry of the field programmable gate array (FPGA) is simplified. The effectiveness of the proposed method is verified through experiments of load fluctuations and supply voltage variations.
\end{abstract}

Keywords: IPMSM, flux weakening operation, FPGA, voltage saturation

\section{Introduction}

Given identical magnetic flux and armature current, an interior permanent magnet synchronous motor (IPMSM), which has a permanent magnet buried inside the rotor core, can produce more torque than a surface permanent magnet synchronous motor (SPMSM) whose magnet is located on the surface of the rotor. This is because IPMSM motors use not only the magnet torque generated between the armature and the magnet, but also reluctance torque caused by magnetic flux between the rotor and the armature. By controlling the armature current vector to weaken magnet flux, IPMSM speeds can be expanded in flux weakening region.

Previous reports discussing current vector control to limit voltage saturation in a flux weakening region ignore the current vector trajectory ${ }^{(1)}$. This method is dependent on a motor's parameters because it needs to utilize an equation which corrects the q-axis inductance on the basis of experimental results. The current vector may change when environmental factors such as temperature, fluctuate. Use of the field programmable gate array (FPGA) for this approach is unsuitable because the formula is too complex.

Kim and Sul describe a method for preventing voltage saturation with a simple voltage regulator ${ }^{(2)}$. This is helpful to researchers because it is not dependent on motor parameters. However, this method requires stringently defined PI parameters for the voltage regulator to avoid voltage saturation.

Jevremovic and Marcetic derived the appropriate PI parameters for the voltage regulator proposed by Kim and Sul using an equation incorporating the features of $\mathrm{d}-\mathrm{q}$ axes current

\footnotetext{
* Shimadzu Corporation

3-9-4, Hikaridai, Seika-cho, Soraku-gun, Kyoto 619-0237, Japan

** Kyushu Institutes of Technology

2-4, Hibikino, Wakamatsu-ku, Kitakyushu, Fukuoka 808-0196, Japan
}

regulation ${ }^{(3)}$. This method assumes q-axis current to be zero and does not clarify whether it is possible to avoid voltage saturation when q-axis current is not zero.

Zhiqian and He describe another method to prevent voltage saturation in the flux weakening region by providing additional hardware to boost the bus voltage ${ }^{(4)}$. Voltage saturation may still occur if the voltage reference exceeds the upper limit. Furthermore, applying this method to existing systems is difficult because it requires additional hardware.

The method proposed by Vaclavek and Blaha finds the intersection of the torque curve and voltage limitation curve on d-q voltage axes first, then converts it to the current vector value on d-q axes ${ }^{(5)}$. It is necessary to avoid the voltage saturation caused by various motor's parameters. All of the above methods focus on controlling the d-axis current. These methods are advantageous with the aspect to shorten start time of an actuator system applied motor as possible so that maximum output torque is generated by tracking the current vector orbit signed by conventional strategies in the flux weakening resion. Also recentlly, since the down-sizing of motor is required with the aspect of instalation features, a copper loss must be reduced less than that of a conventional motor. Namely between to shorten starting time and reducing a loss are trade-off requirement in the end. Though we studied the conditions to satisfy these requirements and to be stable the current vector orbit using conventional methods, it is hard to find out the conditions.

Hence, considering these requirements in mind, we propose a new current vector control method focused on q-axis current. Our method can reduce the copper loss and regulate the PWM modulation rate at $100 \%$ or less, while preventing voltage saturation. The proposed method makes FPGA implementation relatively easy, does not need complicated equations, and does not need to measure bus voltage. The proposed method maintains stable current vector control in flux weakening region even if load and voltage fluctuate. 
Experimental results using IPMSM labware validate our claims.

\section{Current Vector Control in Flux Weakening Region}

2.1 Strategies for Maximum Torque and Current Vector Control in Flux Weakening Region The relationship between voltage $V_{a}$ and current $I_{a}$ at the motor armature is shown in the following equations.

$$
\begin{aligned}
& v_{d c}{ }^{2}+v_{q c}{ }^{2}=V_{a}^{2} \leq V_{\max }{ }^{2} \ldots \ldots \ldots \ldots \ldots \ldots \ldots \ldots \\
& i_{d c}{ }^{2}+i_{q c}{ }^{2}=I_{a}^{2} \leq I_{\max }^{2} \\
& {\left[\begin{array}{c}
v_{d c} \\
v_{q c}
\end{array}\right]=\left[\begin{array}{cc}
R & -\omega_{e} L_{q} \\
\omega_{e} L_{d} & R
\end{array}\right]\left[\begin{array}{c}
i_{d c} \\
i_{q c}
\end{array}\right]+\left[\begin{array}{c}
0 \\
\omega_{e} \phi_{a}
\end{array}\right] \cdots} \\
& T_{m}=\frac{3}{2} P_{n}\left[\phi_{a}+\left(L_{d}-L_{q}\right) i_{d c}\right] i_{q c} \\
& i_{d e x}=\frac{\phi_{a}-\sqrt{\phi_{a}^{2}-8\left(L_{q}-L_{d}\right)^{2} I_{a}^{2}}}{4\left(L_{q}-L_{d}\right)}, \\
& i_{q e x}=\sqrt{I_{a}^{2}-i_{d e x}^{2}} \ldots \ldots \ldots
\end{aligned}
$$

In these equations, $v_{d c}, i_{d c}$, and $L_{d}$ are d-axis components and $v_{q c}, i_{q c}, L_{q}$ are q-axis components. $v_{q c}$ and $v_{d c}$ are the armature voltage; $i_{q c}$ and $i_{d c}$ are the armature current; $L_{d}$ and $L_{q}$ are the armature inductance; $R$ is the armature resistance; $\phi_{a}$ is the permanent magnet flux linkage; and $P_{n}$ is the number of pole pairs.

Equation (1) describes the voltage limit determined by the maximum inverter voltage and the motor speed. The maximum value $V_{\max }$ is determined by the motor wiring type and PWM technique. For example, in the case of a voltage space vector PWM, $V_{\max }$ depends on motor wiring as described by subsequent equations.

$$
\begin{aligned}
& V_{\max }=1 / \sqrt{3} V_{d c} \text { when Motor winding type } \mathrm{Y} \cdots(6-\mathrm{a}) \\
& V_{\max }=\sqrt{3} / 2 V_{d c} \text { when motor winding type } \Delta \cdots(6-\mathrm{b})
\end{aligned}
$$

Equation (2) is a relational expression of the armature current $I_{a}$ and a current limit $I_{\max }$ determined by the allowable temperature rise, the maximum current of the inverter device, the aspect of motor system requirement and so on.

Equation (3) shows the relationship between the current and voltage for IPMSM in the steady state. Equation (4) describes the output torque of the motor as determined by the d-q axes current.

Fig. 1 shows the current limiting Circle $D$ described by equation (2) with radius $I_{\max }$, which is decided in consideration of an environmental condition or system operating conditions, the motor output torque curve $T_{\max }$ by equation (4), and the voltage restriction curve $\omega A, \omega B$, and $\omega C$ which features elliptic curve are calculated using equation (1) and (3) respectively. These elliptic curves signify the current vector orbit which makes best use on the condition of volatage saturation by equation (1). Also the value $\omega C$ represents an operating speed in the flux weakening region. Namely, the operating point $S$ is addressed at the intersection of a given load torque $T L$ and $\omega C$. Curve $O B A$ is a current vector orbit,

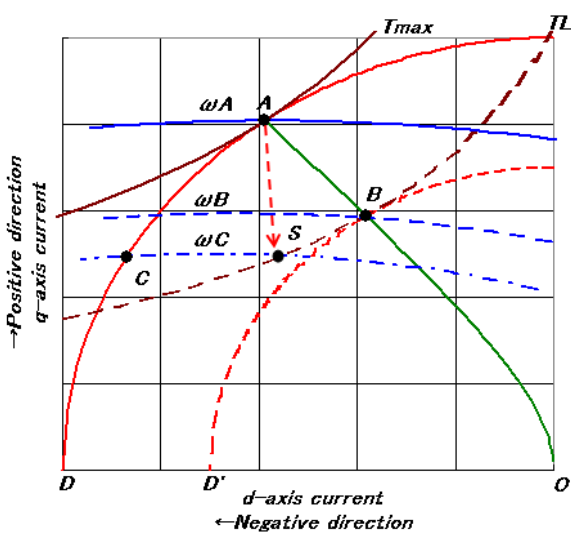

Circle $D$ : maximum current orbit by Eq.(2); Circle $D^{\prime}$ : rated current orbit Tmax: maximum torque trajectory by Eq.(5) $\omega A, \omega B$, and $\omega C$ : the current vector orbit on the condition of voltage saturation.

Fig. 1. d-q current characteristics of IPMSM with torque and speed limitation curves

which is determined by equation (5), to maximize the motor output torque expressed in equation (4). In other words, the maximum output motor torque $T_{\max }$ at point $A$, which is the intersection of curve $O B A$ and Circle $D$, is determined. $T L$ represents the rated load torque when a current vector settle to an operating point $S$. Following the current orbit as dictated by changing current vector in equation (5), when the motor rotates slower than $\omega A$, it operates in the maximum torque region. At this time the current vector is only limited by equation (2), and voltage saturation does not occur, so it is not necessary to take into consideration the voltage restrictions of equation (1).

Consequently, the current vector orbit by equation (5) tracks on the curve $O B A$. In the cases of a conventional current vector strategy in the flux weakening resion, when motor speed is higher than $\omega A$ and come into the flux weakening region, a current vector orbit tracks on Curve $A C$ and settles to the operation point $S$. it is required to implement the suitable current vector strategy to prevent the voltage saturation.

2.2 Current Vector Strategy Focused on d-axis Current Most of the current vector control methods in the flux weakening resion so far, which adjusting d-axis current to decrease permanent magnetic flux as described by equation (4), are advantageous with the aspect to shorten start time of a system applied motor as possible so that maximum output torque is generated by tracking the current vector curve $A C$ by a conventional strategies. But, conventional methods tend to increase a copper loss because of tracking Curve $A C$ approching to operating point $S$. Intuitively, a current vector orbit to reduce a motor loss should be tracked straightforward from point $A$ to point $S$ better than tracking curve $A C$ in Fig. 1. Using this vector orbit, the consideration is whether a response time of the motor speed is within the specified time or not. This is because output torque becomes smaller than that of a conventional method. Namely the trade-off between to shorten starting time and reducing a loss is required in the end. Hence, we studied the configuration proposed by Kim and Sul in Fig. $2^{(2)}$.

1) Whether this configuration could reduce copper loss or not?

As shown in Appendix app.Fig. 1 to app.Fig. 4, it is clearly 
that the current vector orbit does not track straightforward to approach the operating point in flux weakening region. This is undesirable so that it increases a copper loss.

2) Whether the current vector trajectory moves directly and stably towards an operating point $S$, indicated by the arrow in Fig. 1?

So Part II shown in Fig. 2 is the voltage regulator, a propotional and integral which determine the response and the deviation of the voltage regulator of the frequency domain, since a current vector command is set on the basis of this regulator' output, the response of the current vector is also dependent of these parameters.

Therfore, Part II \& Part III regulate and provide a current vector to prevent the voltage saturation with considering the response of the frequency domain, a current regulator, which is higher than that of the voltage regulator typically, is also considered and designed to become the response of the frequency domain fast as possible. The results of some simulated cases are shown in Fig. 3 and Appendix. The results imply that more a proportion gain is set to large, the more a current vector tends to be unstable, and finally it becomes uncontrollable around the point which changes the current vector rapidly such as the point from the maximum torque region into the flux weakening region. Meanwhile, in a steady state, the voltage saturation is prevented and the current vector does not become uncontrollable if proportinal gain is defined appropriately. On the other hand, if the proportional decreases, though a current vector orbit behaves moderatly and becomes stable around the transition point $\mathrm{A}$, this is undesirable because a current respose becomes worse.

We thought "why the current vector is unstable?" as following. When the propotional of current regulator is large to track the current command, which describes the above, the actual current is forced to run over by a current regulator, then it is not able to do a desirable response because the voltage margin is not set to cope with an output of a current regulator owing to regulate at $V_{\text {smax }}$.

We had tried to seek out the cases of both PI regulators which a current vector orbit performs our desirable response. But, it is hard to find out that case.

2.3 Current Vector Strategy Focused on q-axis Current By providing the voltage margin not to saturate, we

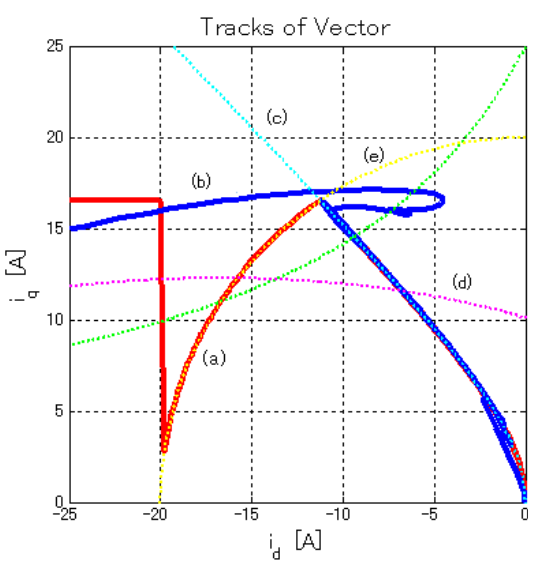

(a) Current command; (b) result; (c) maximum torque trajectory by Eq.(5); (d) voltage limitation by Eq.(1); (e) current limitation by Eq.(2)

Fig. 3. Example of simulation using controller

developed the method able to track the desirable current vector orbit and to be stable even if the current changes rapidly at the transition point.

Fig. 4 represents the proposed motor control system. This system composes an enhanced stability function control and a conventional speed control system. The first part of proposed function compares the actual rate of PWM applied to the motor, $D_{\text {real }}$ equal to $\sqrt{ }\left(v_{q s}{ }^{2}+v_{d s}{ }^{2}\right)$, with the internal PWM criteria of the controller, $D_{\text {crit }}$, in order to prevent voltage saturation.

The second part of the proposed function is the q-axis current limiter which limits maximum torque controller output, $i_{q e x}$, guided by the PWM modulation rate stabilizer. Because of the high gain of the speed regulator, the input current $I_{a}$ will be equal to $I_{\max }$ when actual motor speed is not near the speed command.

Then the maximum torque controller output $i_{d e x}$ becomes to constant value, $i_{d c}$. Assuming the parenthesis in an equation (4) represents an equivalent torque coefficient, it is suggested that motor output Torque, $T_{m}$, is controlled solely by q-axis current, $i_{q c}$. As the motor speed approaches the level dictated by the speed command, $I_{a}$ will decrease below $I_{\max }$. As $i_{d c}$ also reduces, the equivalent torque coefficient inside the parenthesis of equation (4) becomes smaller. The motor will continue to accelerate because output torque exceeds

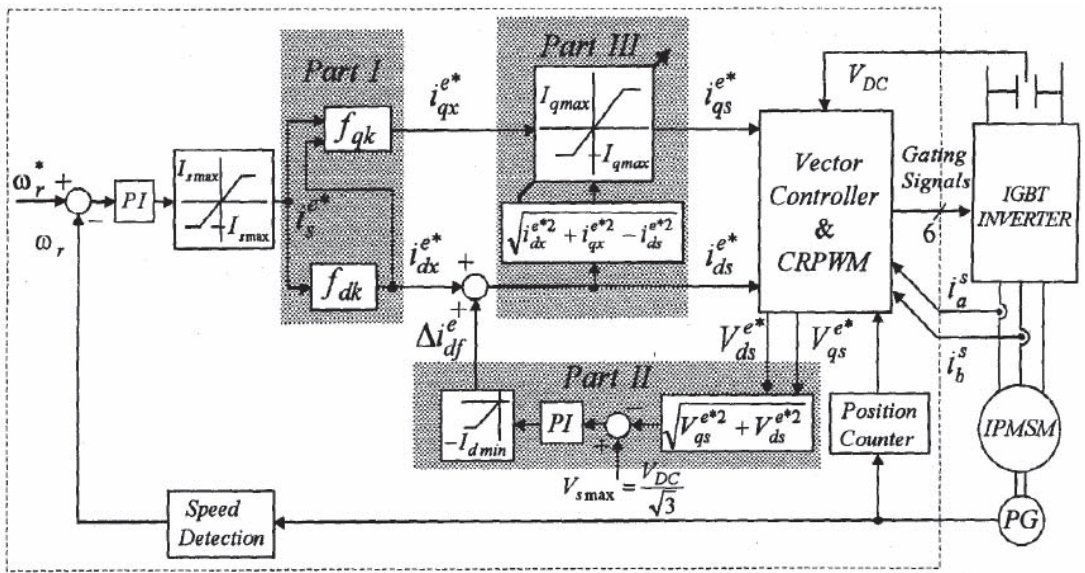

Fig. 2. Current vector controller regulating d-axis current in the flux weakening region ${ }^{(2)}$ 
load torque. Under these operating conditions, $v_{d c}, v_{q c}$, and $D_{\text {real }}$ increase while internal parameter $i_{\text {qlim }}$ acts to decrease the q-axis current $i_{q c}$ to balance the load torque $T L$ as it approaches point $S$ on the load torque curve of Fig. 1.

The PWM rate stabilizer receives signal $D_{\text {real }}$ from the vector controller and produces q-axis current limitation value $i_{\text {qlim }}$ via the following process: First $D_{\text {real }}$ and $D_{\text {crit }}$ are compared, producing value $C R$ which is " +1 " if $D_{\text {real }} \geq D_{\text {crit }}$, and " -1 " if $D_{\text {real }}<D_{\text {crit }}$. The up/down (UD) counter then looks at $C R$ and continuously counts up or down resulting in $D_{f b}$, a value between 0 and 100 representing how degree $D_{\text {real }}$ and $D_{\text {crit }}$ differ. $D_{f b}$ is compared with control value $L R R$, which determines the changing rate of $i_{\text {qlim }}$ at the transition point and transits from the maximum torque region to flux weakening region stably, and the resulting differential, error signal $\varepsilon_{r}$, is delivered to the integrator which produces signal $i_{\text {qlim }}$. In other words, $D_{\text {crit }}$ does an equivalent function of integral and $L R R$ does an equivalent function of proportional respectively.

The function of the PWM rate stabilizer is summarized in Fig. 5, where $D_{\text {crit }}$ set to $95 \%$ and $L R R 30 \%$ for explanation.

When the speed command is given as the operating point $S$ which shows in Fig. 1, the motor output torque is required to overcome the load torque. And the current vector orbit moves along the maximum torque strategy curve. At start-up, voltage saturation does not occur since the actual speed is lower than $\omega A$.

Therefore it is sufficient to consider only the current limit circle $D$. On this condition, since $D_{\text {real }}$ is smaller than $D_{\text {crit }}$, the comparator always output " -1 ", and $D_{f b}$ will reach the lower limit $0 \%$. $\varepsilon_{r}$ becomes $30 \%$, the output $i_{q l i m}$ of the PWM modulation rate stabilizer always keeps the maximum value. Consequently q-axis limiter does not limit the output $i_{q e x}$.

Then the motor accelerates further and close to $\omega A, D_{\text {real }}$ begins to exceed $D_{c r i t}$ and $D_{f b}$ will increases simultaneously. $i_{\text {qlim }}$ is calculated as following.

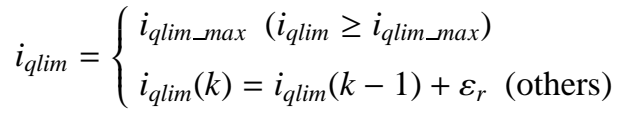

If $D_{f b}>L R R, \varepsilon_{r}<0 . i_{q e x}$ is restricted since $i_{\text {qlim }}$ begins to descend. $L R R$ is index to determine $i_{\text {qlim }}$ response. The motor

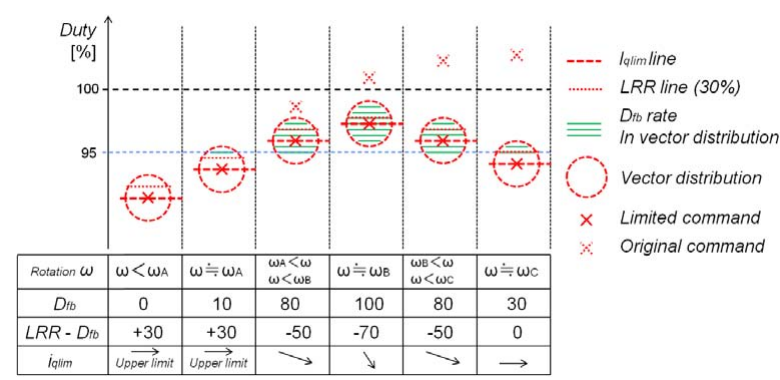

Fig. 5. Behavior of the PWM modulation rate stabilizer

speed continues to accelerate from $\omega A$ to $\omega B$ as torque output is greater than load torque $T L$. At motor speed $\omega B, D_{\text {real }}$ exceeds $D_{c r i t}, D_{f b}$ counts full eventually. As a result, since $\varepsilon_{r}$ becomes a negative steady value, changing rate of $i_{\text {qlim }}$ is also steady from equation (7), and $T m$ decreases. On the condition that the motor speed approaches the vicinity of the operating speed $\omega C, I_{d c}$ begins to descend from $I_{d c \_ \text {max }}$, which is the maximum $d$ axis current generated the maximum torque $T m$. Focusing on q axis current, $i_{q l i m}$ becomes constant value since $D_{f b}$ closes to $L R R$ gradually and $\varepsilon_{r} \approx 0$. Therefore current vector converges to operating point $S$ to balance with $T L$.

\section{Simulation and Experimental Results}

3.1 Simulation Results Fig. 6 shows the step response of the motor speed with current vector trajectory and current wave simulated at point $S$. Speed command is $6,093 \mathrm{~min}^{-1}$, load torque is $2.02 \mathrm{Nm}$, and $V_{d c}$ is $150 \mathrm{~V}$. Parameters of this simulation are shown in Table 1.

As shown in the current vector trajectory of Fig. 6, the simulated motor torque converges on the operating point, but it

Table 1. Simulation conditions

\begin{tabular}{|l|c|}
\hline \multicolumn{1}{|c|}{ The model parameter of IPMSM } & PWM modulation rate's stabilizer \\
\hline Number of pole $: 4$ & \\
Armature resistance $: 0.5[\Omega]$, & \\
d-axis inductance $: 2.07[\mathrm{mH}]$ & Derit $: 95 \%, L R R: 10 \%$ \\
q-axis inductance $: 5.13[\mathrm{mH}]$ & \\
magnet flux linkage $\phi \mathrm{a}: 0.080[\mathrm{~V} \cdot \mathrm{s} / \mathrm{rad}]$ & \\
\hline
\end{tabular}

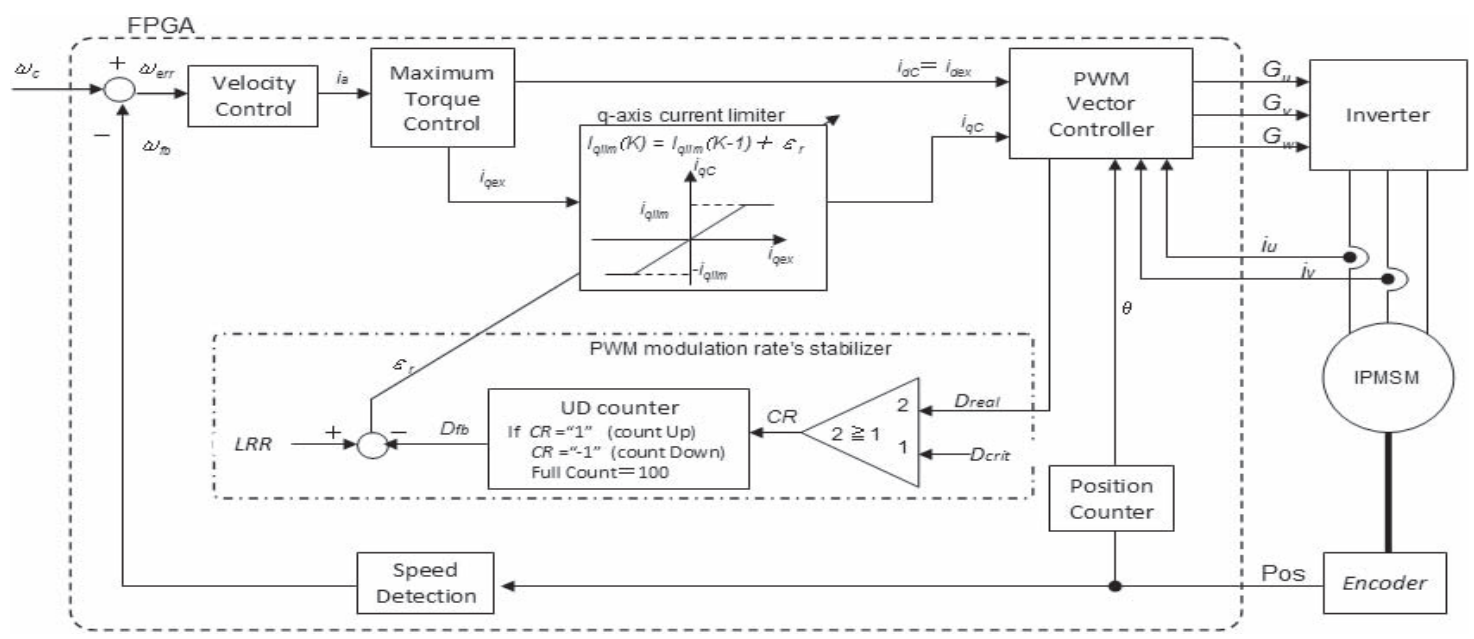

Fig. 4. A proposed current vector controller on the focus of q-axis current in the flux weakening region 


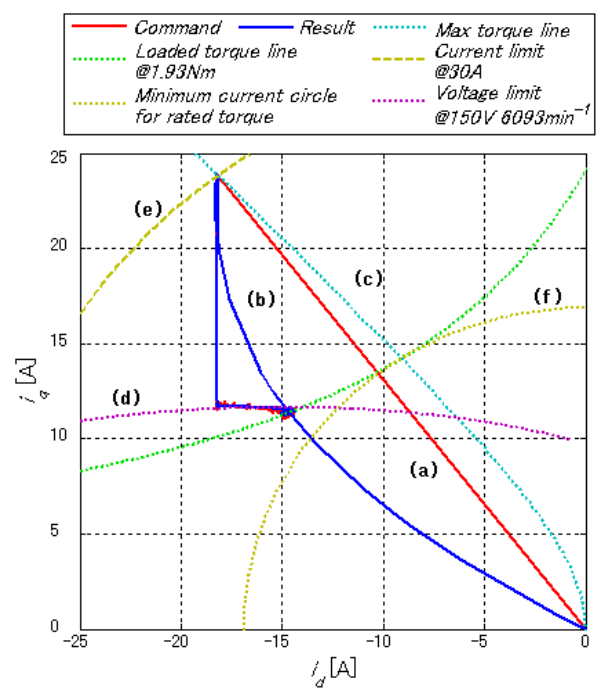

(a) Current command; (b) current result; (c) maximum torque trajectory by Eq.(5); (d) voltage limitation by Eq.(1); (e) current limitation by Eq.(2); (f) minimum current for rated torque

Fig. 6. Simulated trajectory of current vector
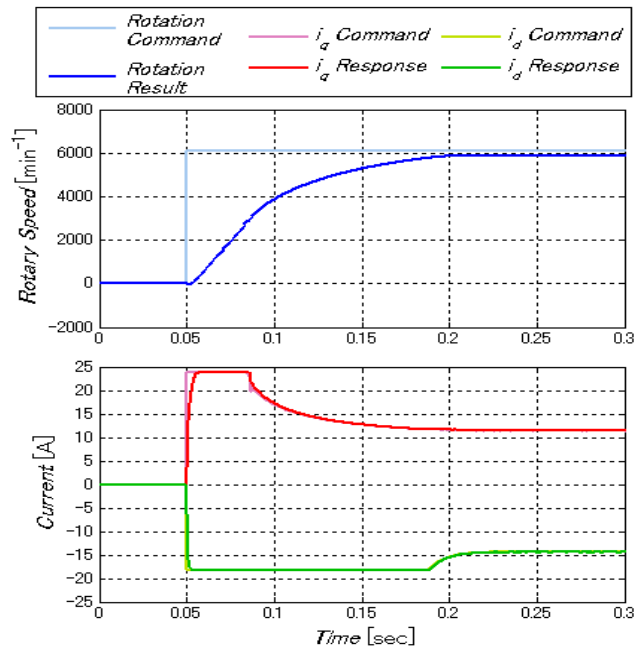

Fig. 7. Simulated step response and d-q axes currents

seems that the simulated rotation has an error to the speed command. The right side of the voltage limitation shown by the equation (1) becomes $0.95 V_{\max }$ since $D_{\text {crit }}$ is set to $95 \%$ shown in Table 1.

As illustrated in Fig. 7, there is no actual error and the motor eventually reaches the reference value. Additionally, without the current vector diverging at the transition point to the field-weakening region, current vector $I_{a}$ follows the current command.

3.2 Experimental Results Fig. 8 shows the labware system for the testing plant. The load to the IPMSM is supplied by controlling the pressure of the hydraulic motor. The load torque control accuracy varies approximately $\pm 10 \%$ from the set value. Speed control, PWM vector function, PWM modulation stabilizer, and q-axis limiter are implemented by the FPGA (Fig. 4). Only the speed command is given externally. Test parameters are the same as those in Table 1.

By the results of simulation and experimence of distributions of actual PWM rate $D_{\text {real }}$ around the transition point $A$,

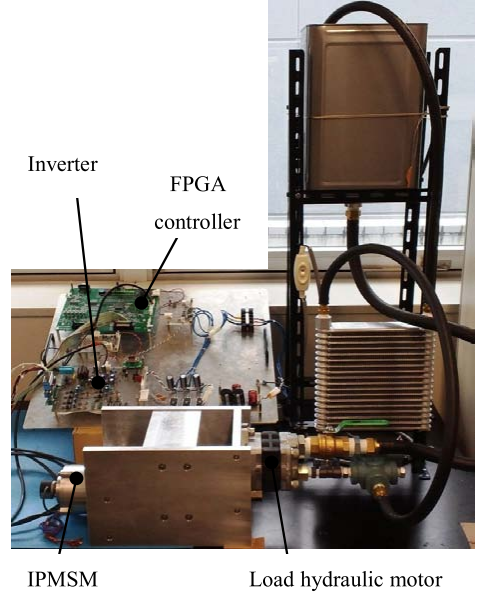

Fig. 8. Labware system

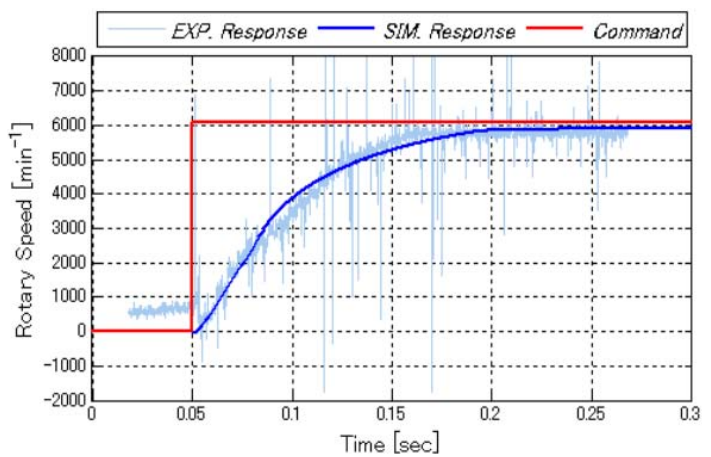

Fig. 9. Step response result of experiment and simulation

in advance,

We decided $D_{\text {crit }}$ is set to $95 \%$. $L R R$ value should be small to make the current vector a more stability in and into the flux weakening region. But the motor output torque also decreases and has an influence on the system start up time if $L R R$ is set too small. Therefore we fixed $L R R 10 \%$ experimentally to satisfy the specified start up time.

\section{Control performance of step response}

Fig. 9 shows the experimental and simulation results of the step response when the speed command changed to the field weakening range $\left(6,093 \mathrm{~min}^{-1}\right)$ from the standstill. Load torque was set to a constant value of $1.93 \mathrm{Nm}$. Rise time to $90 \%$ of reference value is approximately $100 \mathrm{~ms}$ and steady state speed error is within $1 \%$. It seems that the gain of the speed controller has influenced to this error in a steady state. Experimental results are very close to the simulation. The current vector was accurate and stable control was achieved.

\section{Current vector orbit when supply voltage varied}

Fig. 10 shows the current vector orbit when supply voltage varied. The voltage was arbitrarily adjusted between $125 \mathrm{~V}$ and $150 \mathrm{~V}$. To observe the behavior at the transition point to flux weakening region, the speed command and load torque were set to $4,875 \mathrm{~min}^{-1}$ and $1.93 \mathrm{Nm}$, respectively. The voltage limit ellipse at a given constant speed shrinks when the supply voltage is decreased; the current vector trajectory follows where the load torque curve and the voltage limit ellipse intersect. This test exhibited stable current vector control and agreed with the simulation. 

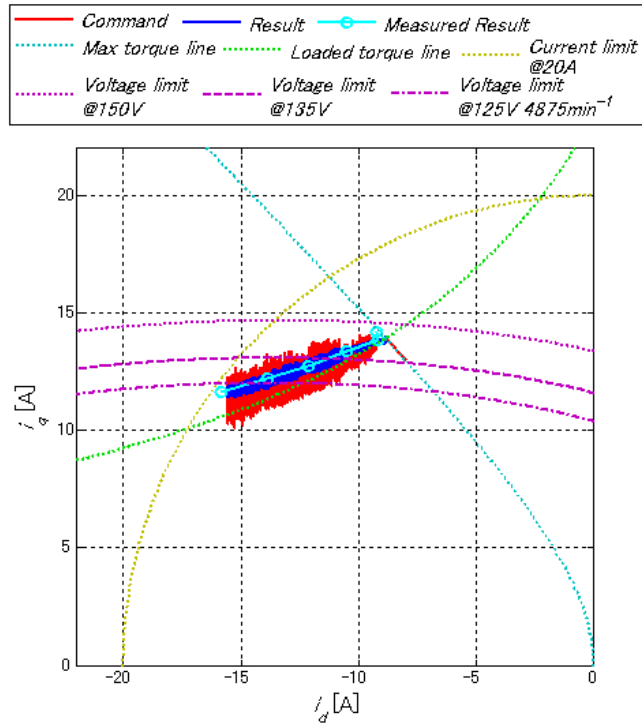

Fig. 10. Vector trajectory on the bus voltage fluctuations

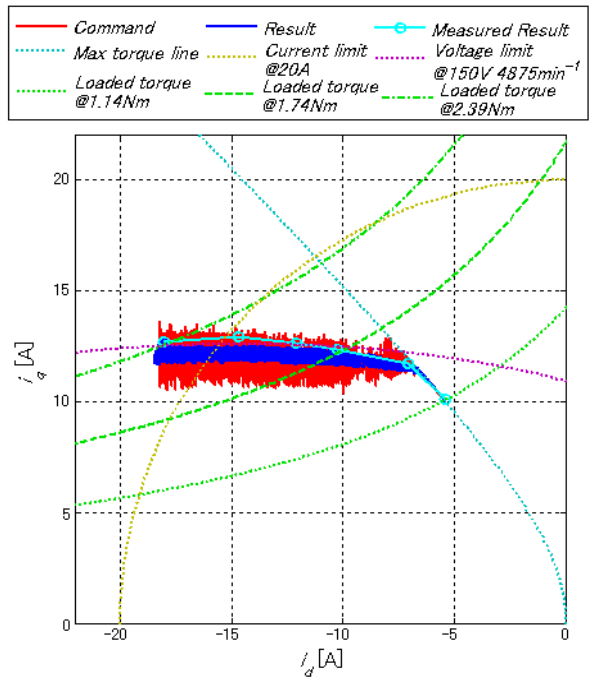

Fig. 11. Vector trajectory on load fluctuations

\section{Current vector orbit when the load torque fluctuates}

Fig. 11 shows the current vector orbit at the transition point to flux weakening region when the load torque is varied between $1.14 \mathrm{Nm}$ and $2.17 \mathrm{Nm}$ with speed command set at $4,875 \mathrm{~min}^{-1}$ and supply voltage at $150 \mathrm{~V}$. When the load torque is small, the current vector orbit aligns with the maximum torque curve. When the load torque curve intersects with the voltage limits ellipse, a stable current vector is achieved. The small torque error between the simulated and experimental results was attributed to the labware torque controller.

\section{Conclusion}

In this paper, we proposed a new method of current vector control to improve stability in and into the field-weakening region. This system prevents voltage saturation by augmenting conventional speed control through q-axis current control using PWM modulation techniques. While conventional systems focus on d-axis current control in flux weakening region, requiring complex square root and multiplier functions to determine the limit value for q-axis current, our system simplifies this to just q-axis current limit control, incorporating a PWM modulation rate stabilizer. The proposed method results in a stable current vector control in flux weakening region. The proposed technique can be implemented in FPGA and can control for fluctuations in voltage and load torque. However, in order to apply this proposed method, the system must have the ability to adequately vary PWM modulation, and the $L R R$ should be chosen carefully for motor specification. In addition, the proposed technique is only applicable to the cases that the d-axis current of the operating point is within the d-axis current which is the intersection of curve $O B A$ and Circle $D$. Future study is required in order to address the above problems.

\section{References}

( 1 ) S. Morimoto, M. Sanada, and Y. Takeda: "Wide-Speed Operation of Interior Permanent Magnet Synchronous Motors with High-Performance Current Regulator", IEEE Trans Ind. Appl., Vol.30, No.4, pp.920-926 (1994)

( 2 ) J.M. Kim and S.K Sul: "Speed Control of Interior Permanent Magnet Synchronous Motor Drive for the Flux Weakening Operation", IEEE Trans Ind. Appl., Vol.33, No.1, pp.43-48 (1997)

( 3 ) V.R. Jevremovic and D.P. Marcetic: "Closed-Loop Flux-Weakening for Permanent Magnet Synchronous Motors", in Proc. 4th IET Conf. on Power Electron., Machines and Drives PEMD, Vol.1, pp.717-721 (2008)

( 4 ) C. Zhiqian and P. He: "The controller of an electric motor drive", The patent journal of Japan, JPA 2010-279176 (2010)

( 5 ) P. Vaclavek and P. Blaha: "Interior Permanent Magnet Synchronous Machine High Speed Operation using Field Weakening Control Strategy", 12th WSEAS International Conf. on SYSTEMS., pp.581-586 (2008)

( 6 ) J.S. Kim and S.K. Sul: "A Novel Voltage Modulation Technique of the Space Vector PWM”, IEEJ Ind. Appl., Vol.116-D, No.8, pp.820-825 (1996)

\section{Appendix}

Study the Configuration Proposed in Fig. 2

We studied the configulation in Fig. 2 by simulating some cases. The simulated results are shown as following. The simulated conditions are same as Table 1 . We thought the PI current regulator is used in the Vector Controller \& CRPWM block shown in Fig. 2, each parameter is shown in the following app.Table 1 . The velocity command used for all casese is the ramp input shown in app.Fig. 7. The results from app.Fig. 1 to app.Fig. 3 are implied that more the propotional

app. Table 1. PI parameter used in the simulations

\begin{tabular}{|c|c|c|c|c|}
\hline Cases & $\begin{array}{c}\text { The conditions } \\
\text { of Part II }\end{array}$ & $\begin{array}{l}\text { The condi } \\
\text { PI current }\end{array}$ & zulator & Remark \\
\hline - & \multirow{5}{*}{$\begin{array}{l}\text { Proportinal : } 0 \\
\text { Integral }: 0.5 \\
\quad * \text { Note } 1\end{array}$} & $\begin{array}{l}\text { Proportiona } \\
\text { Integral }\end{array}$ & $\begin{array}{l}2 \\
300 \\
\end{array}$ & Fig. 3 \\
\hline Case1 & & $\begin{array}{l}\text { Proportiona } \\
\text { Integral }\end{array}$ & $\begin{array}{l}5 \\
0\end{array}$ & Fig.A-1 \\
\hline Case2 & & $\begin{array}{l}\text { Proportiona } \\
\text { Integral }\end{array}$ & $\begin{array}{l}20 \\
0 \\
\end{array}$ & Fig.A-2 \\
\hline Case3 & & $\begin{array}{l}\text { Proportiona } \\
\text { Integral }\end{array}$ & $\begin{array}{l}80 \\
0\end{array}$ & Fig.A-3 \\
\hline Case 4 & & $\begin{array}{l}\text { Proportiona } \\
\text { Integral }\end{array}$ & $\begin{array}{l}20 \\
5\end{array}$ & $\begin{array}{l}\text { Fig.A-4 } \\
\text { Fig.A-5 }\end{array}$ \\
\hline
\end{tabular}

Note 1: When a step response used a proportional only is simulated, the PWM modulation rate is reached at $100 \%$ in a moment. The Part II in Fig. 2 functions at the same moment, $\mathrm{d}$-axis current comes under its influence and is larger toward the negative direction, the motor output torque is lower and the start time draw out consequently. Therefore, integral was only used. 
gain increases, the more the current vector deviates rapidly and is uncontrolable. Next, the results which use PI current regulation are implied that the integral portion contributes to steady-state improvement, but does not contribute to that of the excess state in app.Fig. 4. In addition, the result of time respose in the Case 4 is shown in app.Fig. 5. It shows the zone which motor does not accelerate because the current vector deviates at the transition point.

Lastly, the results of the current vector orbit and the time response are shown in app.Fig. 6 and app.Fig. 7 respectively, using our proposed system, when the same ramp input is inputed to our proposed system and other conditions are the same as Table 1, except a current limit $I_{\max }$ is set to $30 \mathrm{~A}$. The break point on the maximum current orbit is decided by $D_{\text {crit }}$ set in Fig. 4. The different for Fig. 6 is whether it is ramp input or step input.

In ramp input, PWM modulation rate's stabilizer shown in Fig. 4 begins to function at the break point, while the ratio $95 \%$ of $D_{\text {real }}$ for $D_{\text {crit }}$ is maintained, the motor speed nears the specified speed and the current vector also converges in the current to match with load torque.

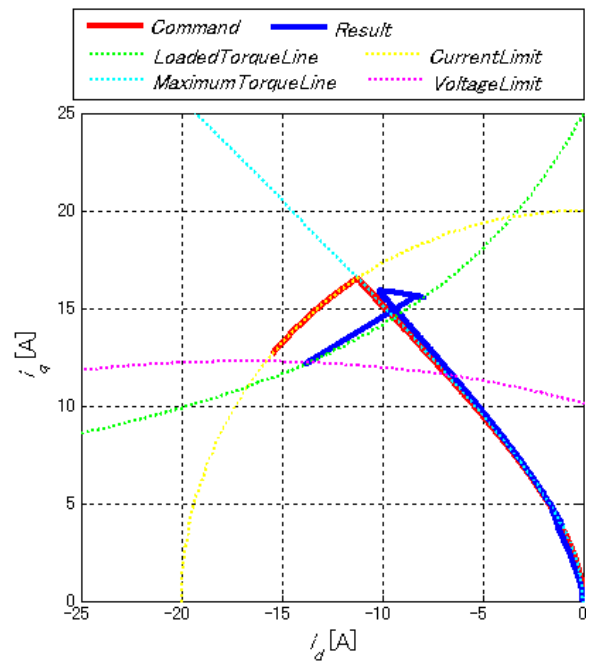

app. Fig. 1. A vector locus simulated by the Case 1

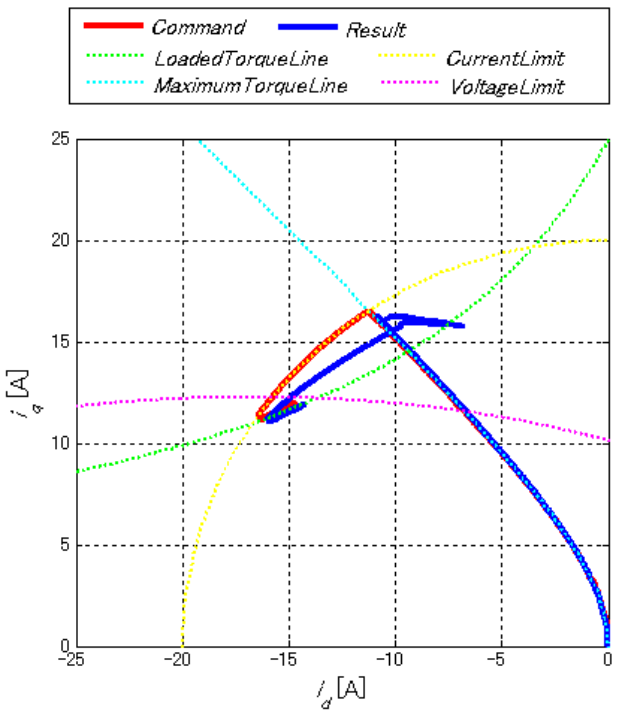

app. Fig. 2. A vector locus simulated by the Case 2

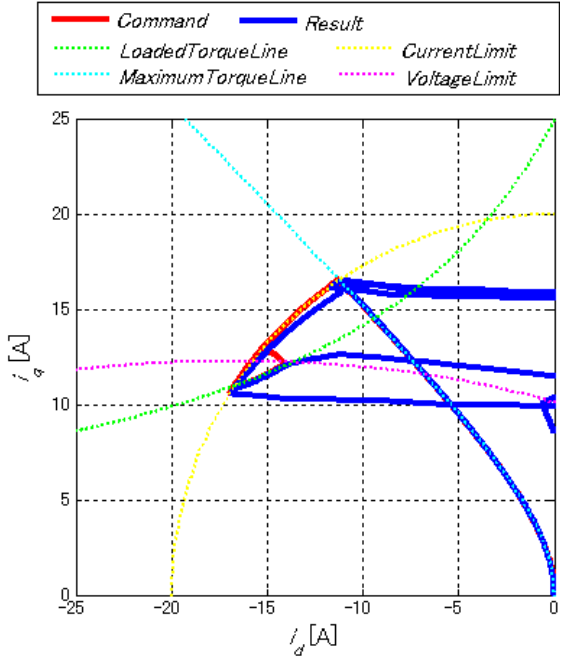

app. Fig. 3. A vector locus simulated by the Case 3

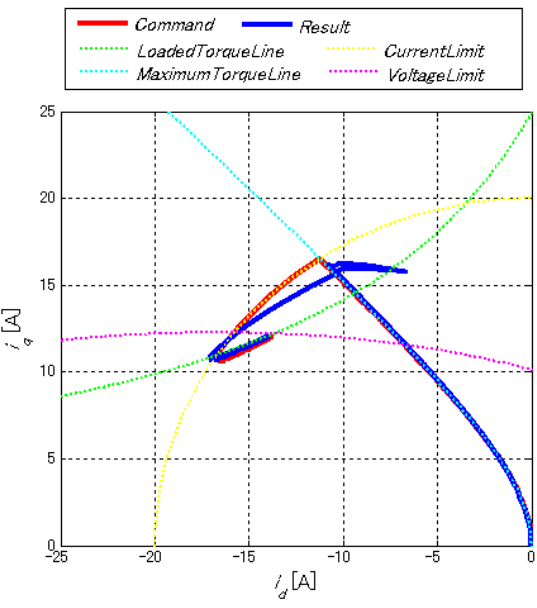

app. Fig. 4. A vector locus simulated by the Case 4

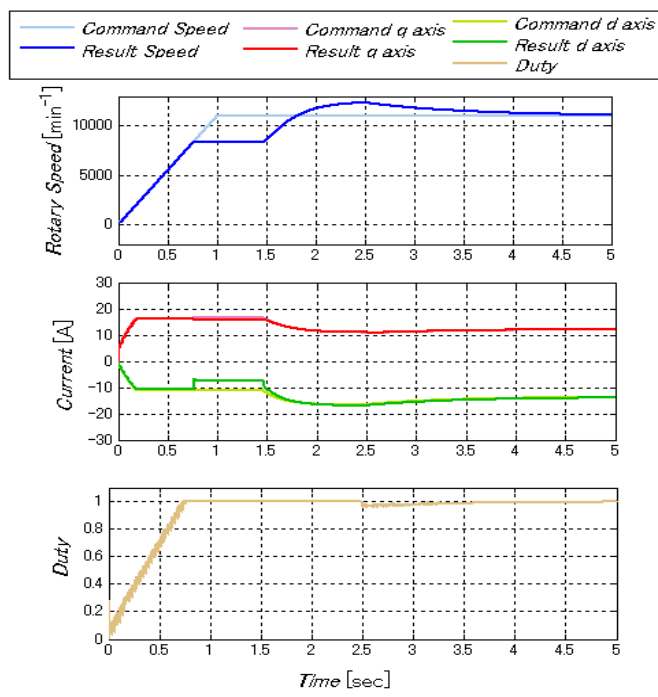

app. Fig. 5. A time response simulated by the Case 4 


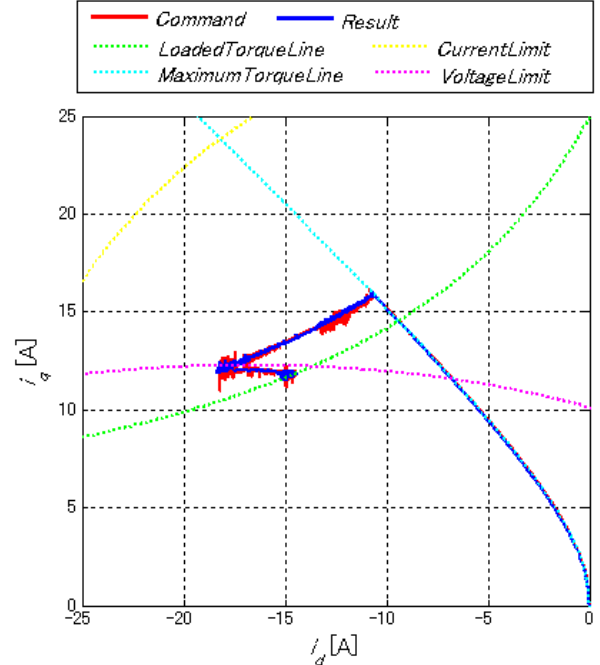

app. Fig. 6. A vector locus for the ramp input
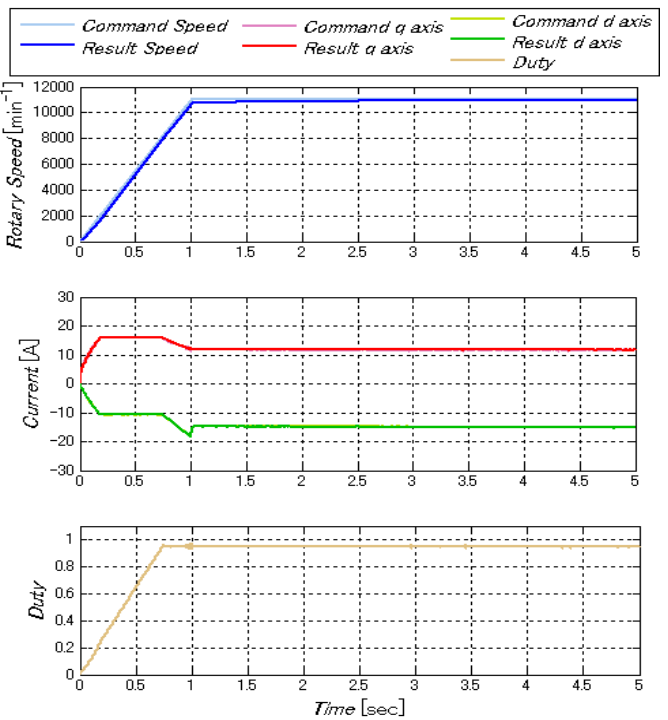

app. Fig. 7. A time response for the ramp input
Yoshihiro Okuyama (Member) He was born in 1963. He received

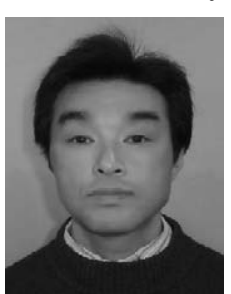
an M.S. degree from Kyushu Institute of Technology, Japan, in 1990 and then joined Shimadzu Corp. His research interests include motor control and power electronics. He is currently enrolled in the doctoral program of the Kyushu Institute of Technology graduate school. He is a member of IEEJ and the Power Electronics Society.

Tomohiro Tanaka (Non-member) He received an M.S. degree from

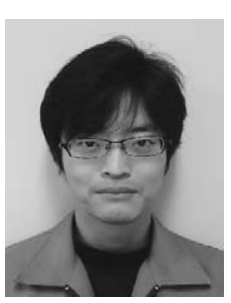
Nagoya University, Japan, in 2010 and then joined Shimadzu Corp. His research interests include motor control and power electronics.

Tsuyoshi Hanamoto (Member) He was born in 1961. He received

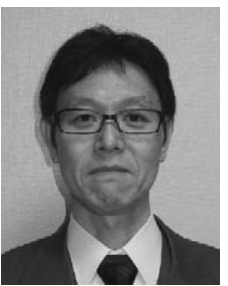
M.S. degree from Kyushu Institute of Technology, Japan, in 1986 and then joined Kobe Works of Kobe Steel, Ltd. In 1990, he joined the Center for Cooperative Research of Kyushu Institute of Technology. From 1997 to 2000 he was with the Department of Electrical Engineering and since April 2000, he has been with the Graduate School of Life Science and Systems Engineering, Kyushu Institute of Technology, where he is presently a Professor. His research interests include motor control and power electronics.

Hiroaki Yamada (Member) He was born in 1979. He received an

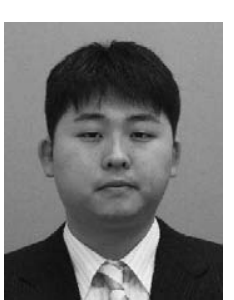
M.E. degree from Shimane University, Shimane in 2004 and a Ph.D. degree from Yamaguchi University, Yamaguchi, in 2007. Beginning in 2007, he was a Lecturer at Kushiro National College of Technology, Hokkaido, Japan. Since 2010, he has held the position of Assistant Professor in the Graduate School of Life Science and Systems Engineering, Kyushu Institute of Technology, Fukuoka, Japan. His current research interest is suppressing the inrush phenomena using PWM converters in power systems and energy harvesting technology. $\mathrm{He}$ is a member of IEEJ, JIPE and IEEE. 